\title{
Computation of Densities and Fluxes of Nonlinear Differential-Difference Equations
}

\author{
By Mark S. Hickman ${ }^{1}$ And Willy A. Hereman ${ }^{2}$ \\ 1 Department of Mathematics and Statistics \\ University of Canterbury \\ Private Bag 4800, Christchurch, New Zealand \\ Email address: M.Hickman@math.canterbury.ac.nz \\ ${ }^{2}$ Department of Mathematical and Computer Sciences \\ Colorado School of Mines \\ Golden CO 80401-1887, USA \\ Email address: whereman@mines.edu
}

\section{Keywords: Conserved Densities; DDEs, Semi-discrete; Lattice; Nonlinear}

Chains; Miura Map

Direct methods to find conserved densities and fluxes of differential-difference equations are presented and illustrated for the Kac-van Moerbeke and modified Volterra lattices. A Miura map which connects both lattices is explicitly constructed based on homotopic deformation. The map is used recursively to compute conserved densities of the Kac-van Moerbeke lattice. The algorithms presented could be implemented in computer algebra systems and could be used to investigate the integrability of semi-discrete lattices.

\section{Introduction}

Dating back to the work of Fermi, Pasta, and Ulam in the 1950's (Fermi et al. 1965) differential-difference equations (DDEs) have been the focus of many nonlinear studies. A number of physically interesting problems can be modeled with nonlinear DDEs, including particle vibrations in lattices, currents in electrical networks, pulses in biological chains, etc. DDEs play in important role in queuing problems and discretizations in solid state and quantum physics. Last but not least, they are used in numerical simulations of nonlinear PDEs.

Recently, there has been a renewed interest in DDEs (see for example Teschl 2000 for a literature review). DDEs are semi-discretized because only the space variable is discretized while time is kept continuous. This is in contrast to their fully discretized counterparts, called difference equations, which are currently extensively studied (see for example Clarkson \& Nijhoff 1999, Hietarinta et al. 2001, Levi \& Ragnisco 2000, Levi et al. 1996).

In this paper we focus on one aspect of the integrability of DDEs, namely the computation of conserved densities and associated fluxes (of a given form) via direct 
methods which could be implemented in computer algebra systems line MAPLE, MATHEMATICA, and MUPAD.

The main result of this paper is three-fold.

(1) Two novel methods to construct families of conserved densities are presented. The first method requires solving the integrability condition for the unknown density. Surprisingly, that integrability condition is quite amenable to analysis and a closed-form solution can be found. The solution method resembles the solution technique for solving the defining equations encountered in the computation of point symmetries of PDEs (Hereman 1996).

(2) The second method is more algorithmic and primarily useful to find fluxes. It uses repeated decomposition of the identity operator I to find pieces in and outside the image $(\mathrm{Im})$ of the operator $\Delta=\mathrm{D}-\mathrm{I}$, where $\mathrm{D}$ is the up-shift operator.

(3) Third, a homotopic deformation of the KvM lattice is constructed explicitly. The Miura map (discrete analogue of the transformation (given in Miura 1968) which connects the KvM and the $\mathrm{mV}$ lattices follows as a special case. Similar to the strategy given in Miura et al. 1968, using the Miura map recursively one can generate densities of the KvM lattice order by order.

Miura maps, in connection with local conservation laws, allow one to construct master symmetries. In turn, based on master symmetries one can find conserved densities and build new integrable DDEs (Cherdanstsev \& Yamilov 1995, 1996).

The techniques described in this paper are also applicable to nonlinear systems of DDEs. Yet, to keep the ideas transparent and to avoid lengthy computations (which are best performed with computer algebra systems), we use the scalar Kacvan Moerbeke (KvM) (Kac \& van Moerbeke 1975) and modified Volterra (mV) (Adler et al. 1999, Cherdanstsev \& Yamilov 1995) lattices as leading examples.

Strategies and MATHEMATiCA code to automatically compute polynomial densities and generalized symmetries of DDEs can be found in Göktaş \& Hereman 1998, 1999, Göktaş et al. 1997 and Hereman et al. 1998. In contrast, the methods in the present paper are no longer restricted to polynomial densities and fluxes. For computer algebra algorithms related to integrability testing based on master symmetries we refer to Oevel et al. 1989.

There is a vast body of work on DDEs, including investigations of integrability criteria, the computation of densities, generalized and master symmetries, recursion operators, etc. Notably is work by Levi and colleagues (Levi \& Ragnisco 1978, Levi \& Yamilov 1997), Yamilov (Yamilov 1993, 1994) and co-workers (Adler et al. 1999, Cherdantsev \& Yamilov 1995, 1996, Shabat \& Yamilov 1991, 1997, Svinolupov \& Yamilov 1991), where the classification of DDEs (into canonical forms), integrability tests, and connections between integrable PDEs and DDEs are analyzed in detail. To a large extend, the classification and integrability testing of discrete equations mimics that of continuous equations (for reviews and references consult Adler et al. 2000, Mikhailov et al. 1987, 1990, Sokolov \& Shabat 1984).

A wealth of information about integrable DDEs can be found in papers by Suris (Suris 1997a, 1997b, 1999, 2001) and his book (Suris 2002) in progress. Suris and others have shown that many lattices are closely related to the celebrated Toda lattice (Toda 1989), its relativistic counterpart due to Ruijsenaars (Ruijsenaars 1990), the KvM lattice, and the two-component Volterra system (Shabat \& Yamilov 1991, 1997). 
Our paper is organized as follows. Section 2 covers preliminary material about conservation laws of DDEs. The KvM lattice exemplifies the introductory notions and notation. In analogy to a result for PDEs, the discrete Euler operator (or variational derivative) is introduced as a valuable tool to test conserved densities. We proof the necessary and sufficient condition for a function of a discrete variable (and its shifts) to be the total difference of another function of discrete variables. The section concludes with an application of the Euler operator to verify a density of the KvM lattice.

Using the discrete Euler operator we outline a strategy to compute conserved densities of nonlinear lattices in Section 3. Details of the computation of a family of densities are given for the $\mathrm{mV}$ lattice.

In Section 4 we focus on the computation of fluxes. We give a direct procedure to compute densities as well as fluxes. We revisit the $\mathrm{mV}$ lattice to illustrate the method.

In Section 5 we construct the discrete version of the Miura map, which links the $\mathrm{mV}$ and the KvM lattices. In analogy with the continuous case, we use the map recursively to generate the densities for the KvM lattice.

We draw some conclusions in Section 6.

\section{Differential-Difference Equations}

In this section we review preliminary material about densities and fluxes of differentialdifference equations.

Consider a nonlinear (autonomous) DDE of the form

$$
\frac{d}{d t} u_{n}=f\left(u_{n-l}, u_{n-l+1}, \ldots, u_{n}, \ldots, u_{n+m-1}, u_{n+m}\right)
$$

with

$$
\frac{\partial f}{\partial u_{n-l}} \frac{\partial f}{\partial u_{n+m}} \neq 0 \text {, }
$$

where $n$ is an arbitrary integer. In general, $f$ is a vector-valued function of a finite number of dynamical variables and each $u_{k}$ is a vector-valued function of $t$.

The index $n$ may lie in $\mathbf{Z}$, or the $u_{k}$ may be periodic, $u_{k}=u_{k+N}$. The integers $l$ and $m$ measure the degree of non-locality in (2.1). If $l=m=0$ then the equation is local and reduces to a system of ordinary differential equations.

The (up-)shift operator D is defined by

$$
\mathrm{D} u_{k}=u_{k+1} \text {. }
$$

Its inverse, called the down-shift operator, is given by $\mathrm{D}^{-1} u_{k}=u_{k-1}$. Obviously, $u_{k}=\mathrm{D}^{k} u_{0}$. The action of $\mathrm{D}$ and $\mathrm{D}^{-1}$ is extended to functions by acting on their arguments. For example,

$$
\mathrm{D} g\left(u_{p}, u_{p+1}, \ldots, u_{q}\right)=g\left(\mathrm{D} u_{p}, \mathrm{D} u_{p+1}, \ldots, \mathrm{D} u_{q}\right)=g\left(u_{p+1}, u_{p+2}, \ldots u_{q+1}\right) .
$$

In particular,

$$
\mathrm{D}\left(\frac{\partial}{\partial u_{k}} g\left(u_{p}, u_{p+1}, \ldots, u_{q}\right)\right)=\frac{\partial}{\partial u_{k+1}} g\left(u_{p+1}, u_{p+2}, \ldots, u_{q+1}\right) .
$$


Moreover, for equations of type (2.1), the shift operator commutes with the time derivative

$$
\begin{aligned}
\mathrm{D}\left(\frac{d}{d t} u_{n}\right) & =\mathrm{D} f\left(u_{n-l}, u_{n-l+1}, \ldots, u_{n}, \ldots, u_{n+m-1}, u_{n+m}\right) \\
& =f\left(u_{n-l+1}, u_{n-l+2}, \ldots, u_{n+1}, \ldots, u_{n+m}, u_{n+m+1}\right) \\
& =\frac{d}{d t} u_{n+1} \\
& =\frac{d}{d t}\left(\mathrm{D} u_{n}\right) .
\end{aligned}
$$

Thus, with the use of the shift operator, the entire system (2.1) which may be an infinite set of ordinary differential equations is generated from a single equation

$$
\frac{d}{d t} u_{0}=f\left(u_{-l}, u_{-l+1}, \ldots, u_{0}, \ldots, u_{m-1}, u_{m}\right)
$$

with

$$
\frac{\partial f}{\partial u_{-l}} \frac{\partial f}{\partial u_{m}} \neq 0
$$

Next, we define the (forward) difference operator, $\Delta=\mathrm{D}-\mathrm{I}$, by

$$
\Delta u_{k}=(\mathrm{D}-\mathrm{I}) u_{k}=u_{k+1}-u_{k},
$$

where I is the identity operator. This operator takes the role of a spatial derivative on the shifted variables as many examples of DDEs arise from discretization of a PDE in $(1+1)$ variables (Shabat \& Yamilov 1988). The difference operator clearly extends to functions, that is

$$
\Delta g\left(u_{p}, u_{p+1}, \ldots, u_{q}\right)=g\left(u_{p+1}, u_{p+2}, \ldots, u_{q+1}\right)-g\left(u_{p}, u_{p+1}, \ldots, u_{q}\right) .
$$

For any function $g=g\left(u_{p}, u_{p+1}, \ldots, u_{q}\right)$, the total time derivative $\mathrm{D}_{t} g$ is computed as

$$
\begin{aligned}
\mathrm{D}_{t} g & =\sum_{k=p}^{q} \frac{\partial g}{\partial u_{k}} \frac{d}{d t} u_{k} \\
& =\left(\sum_{k=p}^{q} \frac{\partial g}{\partial u_{k}} \mathrm{D}^{k}\right) \frac{d}{d t} u_{0} \\
& =\left(\sum_{k=p}^{q} \frac{\partial g}{\partial u_{k}} \mathrm{D}^{k}\right) f\left(u_{-l}, u_{-l+1}, \ldots, u_{m}\right)
\end{aligned}
$$

on solutions of (2.1). A simple calculation shows that the shift operator D commutes with $\mathrm{D}_{t}$, and so do $\mathrm{D}$ and $\Delta$.

A function $\rho=\rho\left(u_{p}, u_{p+1}, \ldots, u_{q}\right)$ is a (conserved) density of (2.2) if there exists a function $J=J\left(u_{r}, u_{r+1}, \ldots, u_{s}\right)$, called the (associated) flux, such that

$$
\mathrm{D}_{t} \rho+\Delta J=0
$$

is satisfied on the solutions of (2.2). Eq. (2.3) is called a local conservation law. 
Any shift of a density is trivially a density since

$$
\mathrm{D}_{t} \mathrm{D}^{k} \rho+\Delta \mathrm{D}^{k} J=\mathrm{D}^{k}\left(\mathrm{D}_{t} \rho+\Delta J\right)=0,
$$

with associated flux $\mathrm{D}^{k} J$.

Constants of motion for (2.2) are easily obtained from a densities and their shifts. Indeed, for any density $\rho$ with corresponding flux $J$, consider

$$
\Omega=\sum_{k=p}^{q} \mathrm{D}^{k} \rho
$$

The total time derivative of $\Omega$ is

$$
\begin{aligned}
\mathrm{D}_{t} \Omega & =-\sum_{k=p}^{q} \Delta \mathrm{D}^{k} J \\
& =-\sum_{k=p}^{q}\left(\mathrm{D}^{k+1}-\mathrm{D}^{k}\right) J \\
& =-\left(\mathrm{D}^{q+1}-\mathrm{D}^{p}\right) J .
\end{aligned}
$$

Applying appropriate boundary conditions (e.g. all $u_{k} \rightarrow 0$, as $k \rightarrow \pm \infty$ ) one gets the conservation law

$$
\mathrm{D}_{t}\left(\sum_{k=-\infty}^{\infty} \mathrm{D}^{k} \rho\right)=\lim _{q \rightarrow \infty} \mathrm{D}^{q+1} J-\lim _{p \rightarrow-\infty} \mathrm{D}^{p} J=0 .
$$

For a periodic chain where $u_{k}=u_{k+N}$, after summing over a period, one obtains

$$
\mathrm{D}_{t}\left(\sum_{k=0}^{N} \mathrm{D}^{k} \rho\right)=\mathrm{D}^{N+1} J-\mathrm{D}^{0} J=J-J=0 .
$$

In either case, $\Omega$ is a constant of motion of (2.2) since $\Omega$ does not change with time.

A density which is a total difference,

$$
\rho=\Delta F
$$

(so that $\mathrm{D}_{t} \rho=\Delta \mathrm{D}_{t} F$ and therefore $J=-\mathrm{D}_{t} F$ is an associated flux), is called trivial. These densities lead to trivial conservation laws since

$$
\Omega=\sum_{k=p}^{q} \mathrm{D}^{k} \Delta F=\mathrm{D}^{q+1} F-\mathrm{D}^{p} F
$$

holds identically (not just on solutions of (2.2)).

Two densities $\rho, \tilde{\rho}$ are called equivalent if $\rho-\tilde{\rho} \in \operatorname{Im} \Delta$, i.e. $\rho-\tilde{\rho}=\Delta F$ for some $F$. Equivalent densities, denoted by $\rho \sim \tilde{\rho}$, differ by a trivial density and yield the same conservation law. Also, $\rho \sim D^{k} \rho$, and (2.4) expresses that $\rho \sim 0$.

To illustrate the above notions we now turn to a simple example. 
Example: The Kac-van Moerbeke lattice

Consider the Kac-van Moerbeke (KvM) lattice (Kac \& van Moerbeke 1975, Manakov 1975, Moser 1975),

$$
\frac{d u_{n}}{d t}=u_{n}\left(u_{n+1}-u_{n-1}\right)
$$

or, equivalently,

$$
\frac{d u_{0}}{d t}=u_{0}\left(u_{1}-u_{-1}\right)
$$

Eq. (2.6) is often referred to as a Volterra lattice (Volterra 1931, Yamilov 1994), although it is a special case of the two-component Volterra system (Hirota \& Satsuma 1976, Shabat \& Yamilov 1991).

Lattice (2.6) arises in the study of Langmuir oscillations in plasmas, population dynamics, quantum field theory, polymer science, and appears in the context of matrix factorizations (see references in Teschl 2000).

The KvM lattice (2.5) appears in the literature in various forms, including

$$
\frac{d R_{n}}{d t}=\frac{1}{2}\left(e^{-R_{n-1}}-e^{-R_{n+1}}\right)
$$

and

$$
\frac{d w_{n}}{d t}=w_{n}\left(w_{n+1}^{2}-w_{n-1}^{2}\right) .
$$

Both are related to (2.5) by simple transformations (Teschl 2000).

The function

$$
\rho=u_{0}
$$

is a density for (2.6) since

$$
\begin{aligned}
\mathrm{D}_{t} \rho & =\left(\frac{\partial}{\partial u_{0}} \rho\right) \frac{d}{d t} u_{0} \\
& =u_{0}\left(u_{1}-u_{-1}\right) \\
& =\Delta\left(u_{0} u_{-1}\right)
\end{aligned}
$$

with associated flux $J=-u_{0} u_{-1}$. Thus $\rho_{k}=\mathrm{D}^{k} \rho=u_{k}$ are also densities with associated fluxes $J_{k}=\mathrm{D}^{k} J=-u_{k} u_{k-1}$. Therefore,

$$
\Omega=\sum_{k=p}^{q} \mathrm{D}^{k} \rho=\sum_{k=p}^{q} u_{k},
$$

and

$$
\begin{aligned}
\mathrm{D}_{t} \Omega & =\sum_{k=p}^{q} \Delta\left(u_{k} u_{k-1}\right) \\
& =\sum_{k=p}^{q} u_{k+1} u_{k}-u_{k} u_{k-1} \\
& =u_{q+1} u_{q}-u_{p} u_{p-1} \\
& =-\left(\mathrm{D}^{q+1}-\mathrm{D}^{p}\right) J .
\end{aligned}
$$

Article submitted to Royal Society 
Applying appropriate boundary conditions we get the conservation law

$$
\mathrm{D}_{t}\left(\sum_{k=-\infty}^{\infty} u_{k}\right)=\lim _{q \rightarrow \infty} u_{q+1} u_{q}-\lim _{p \rightarrow-\infty} u_{p} u_{p-1}=0 .
$$

Eq. (2.6) has infinitely many couples $\rho$ and $J$. The above computations could be repeated for other conserved densities, such as $\rho=u_{0}\left(u_{0}+2 u_{1}\right)$ and $\rho=u_{0}\left(u_{0}^{2}+\right.$ $\left.3 u_{1}\left(u_{0}+u_{1}+u_{2}\right)\right)$ listed in Göktaş et al. 1997.

We now introduce a tool to test whether or not a function is a total difference.

The discrete Euler operator (variational derivative)

It is well-known that a (continuous) function $g\left(u(x), u^{\prime}(x), u^{\prime \prime}(x), \ldots, u^{(n)}(x)\right)$ can be integrated with respect to $x$ if and only if (iff) the variational derivative of $g$ vanishes. Formally, $g=\mathrm{D}_{x} h$ for some function $h\left(u(x), u^{\prime}(x), u^{\prime \prime}(x), \ldots, u^{(m)}(x)\right)$ iff $E(g)=0$. Here, $\mathrm{D}_{x}$ refers to total differentiation with respect to $x$ and $E$ is the continuous Euler operator (variational derivative).

In particular, a function $\rho$ is a conserved density of a PDE iff $\mathrm{D}_{t} \rho$ is in the kernel (Ker) of the Euler operator (see for example Göktaş \& Hereman 1997). We now present a discrete analogue of this important result.

A function $g=g\left(u_{p}, u_{p+1}, \ldots, u_{q}\right), p, q \in \mathbf{Z}$, is a total difference if there exists another function $h=h\left(u_{p}, u_{p+1}, \ldots, u_{q-1}\right)$, such that $g=\Delta h=(\mathrm{D}-\mathrm{I}) h$.

A necessary and sufficient condition for a function $g$ to be a total difference is that

$$
\mathrm{E}(g)=0
$$

where $\mathrm{E}$ is the discrete Euler operator (variational derivative) (Adler et al. 1999, Shabat \& Yamilov 1991) defined by

$$
\mathrm{E}(g)=\sum_{k=p}^{q} \mathrm{D}^{-k}\left(\frac{\partial}{\partial u_{k}} g\right) .
$$

Note that we can rewrite the Euler operator as

$$
\mathrm{E}(g)=\frac{\partial}{\partial u_{0}}\left(\sum_{k=p}^{q} \mathrm{D}^{-k} g\right)
$$

and that

$$
\sum_{k=p}^{q} \mathrm{D}^{k} \Delta=\mathrm{D}^{q+1}-\mathrm{D}^{p}
$$

We first show that the condition (2.9) is necessary.

Suppose that $g=\Delta h$ with $h=h\left(u_{p}, u_{p+1}, \ldots, u_{q-1}\right)$ then

$$
\begin{aligned}
\mathrm{E}(\Delta h) & =\frac{\partial}{\partial u_{0}}\left(\sum_{k=p}^{q} \mathrm{D}^{-k} \Delta h\right) \\
& =\frac{\partial}{\partial u_{0}}\left(\mathrm{D}^{-p+1} h-\mathrm{D}^{-q} h\right) \\
& =0
\end{aligned}
$$


since neither $\mathrm{D}^{-p+1} h=h\left(u_{1}, u_{2}, \ldots, u_{q-p}\right)$ nor $\mathrm{D}^{-q} h=h\left(u_{p-q}, u_{p-q+1}, \ldots, u_{-1}\right)$ depend on $u_{0}$. Thus, if $g$ is a total difference then $\mathrm{E}(g)=0$.

Conversely, to prove that (2.9) is sufficient, suppose that $\mathrm{E}(g)=0$ holds. Without loss of generality, we can set $p=0$ since we can replace $g$ by $\mathrm{D}^{-p} g$ (and relabel $q-p$ by $q)$. Thus, for $g=g\left(u_{0}, u_{1}, \ldots, u_{q}\right)$ we have

$$
\begin{aligned}
0 & =\frac{\partial}{\partial u_{q}}(\mathrm{E}(g)) \\
& =\frac{\partial^{2}}{\partial u_{q} \partial u_{0}}\left(\sum_{k=0}^{q} \mathrm{D}^{-k} g\right) \\
& =\frac{\partial^{2}}{\partial u_{0} \partial u_{q}} g
\end{aligned}
$$

since no other term will depend on $u_{q}$. Thus,

$$
\begin{aligned}
g\left(u_{0}, u_{1}, \ldots, u_{q}\right) & =\tilde{g}^{(1)}\left(u_{0}, u_{1}, \ldots, u_{q-1}\right)+g^{(2)}\left(u_{1}, \ldots, u_{q}\right) \\
& =g^{(1)}\left(u_{0}, u_{1}, \ldots, u_{q-1}\right)-g^{(2)}\left(u_{0}, u_{1}, \ldots, u_{q-1}\right)+g^{(2)}\left(u_{1}, u_{2}, \ldots, u_{q}\right) \\
& =g^{(1)}\left(u_{0}, u_{1}, \ldots, u_{q-1}\right)+\Delta g^{(2)}\left(u_{0}, u_{1}, \ldots, u_{q-1}\right)
\end{aligned}
$$

for some functions $g^{(1)}$ and $g^{(2)}$. Therefore,

$$
0=\mathrm{E}(g)=\mathrm{E}\left(g^{(1)}\right)+\mathrm{E}\left(\Delta g^{(2)}\right)=\mathrm{E}\left(g^{(1)}\right)
$$

by (2.12). Repeating this procedure on $g^{(1)}$, we can write $g^{(1)}$ as a function of the $q-1$ variables $u_{0}, u_{1}, \ldots, u_{q-2}$ plus a total difference. We eventually obtain

$$
g\left(u_{0}, u_{1}, \ldots, u_{q}\right)=K\left(u_{0}\right)+\Delta G\left(u_{0}, u_{1}, \ldots, u_{q-1}\right),
$$

where $\Delta G$ is the sum of all the total difference terms and with

$$
0=\mathrm{E}(g)=\mathrm{E}(K)=\frac{\partial K}{\partial u_{0}} .
$$

Hence, $K$ is a constant. Let $\varphi$ be the unit "constant" shifted function; that is $\mathrm{D}^{k} \varphi=k$ and so $\Delta \varphi=1$. Thus $K=K \Delta \varphi$ and so $g$ is a total difference,

$$
g=\Delta(G+K \varphi) .
$$

The integration of (2.13), resulting in (2.14) is important. Indeed, if (2.13) is satisfied then separation of variables leads to a total difference. This result will be used on several occasions later in the paper.

\section{Application of the Euler operator}

The discrete Euler operator (2.10) is a most valuable tool to test conserved densities. Indeed, $\rho$ is a density of $(2.2)$ iff $\mathrm{D}_{t} \rho \in \operatorname{Ker} E$ i.e.

$$
\mathrm{E}\left(\mathrm{D}_{t} \rho\right)=0
$$

Moreover, $\rho$ is a non-trivial density if, in addition, $\mathrm{E}(\rho) \neq 0$. If $(2.15)$ holds, then $\mathrm{D}_{t} \rho \in \operatorname{Im} \Delta$. This implies the existence of a $J$ for which (2.3) is satisfied. 
For the example of the KvM lattice (2.6), $\rho=2 u_{0} u_{1}+u_{1}^{2}$ is a density since

$$
\begin{aligned}
\mathrm{E}\left(\mathrm{D}_{t} \rho\right)= & \mathrm{E}\left(\mathrm{D}_{t}\left(2 u_{0} u_{1}+u_{1}^{2}\right)\right) \\
= & 2 \mathrm{E}\left(u_{1} u_{0}\left(u_{1}-u_{-1}\right)+\left(u_{0}+u_{1}\right) u_{1}\left(u_{2}-u_{0}\right)\right) \\
= & -2 \mathrm{E}\left(u_{0} u_{-1} u_{1}-u_{0} u_{1} u_{2}+u_{0}^{2} u_{1}-u_{1}^{2} u_{2}\right) \\
= & -2\left(\mathrm{D}\left(u_{0} u_{1}\right)+\mathrm{D}^{0}\left(u_{-1} u_{1}-u_{1} u_{2}+2 u_{0} u_{1}\right)+\mathrm{D}^{-1}\left(u_{0} u_{-1}-u_{0} u_{2}+u_{0}^{2}-2 u_{1} u_{2}\right)\right. \\
& \left.-\mathrm{D}^{-2}\left(u_{0} u_{1}+u_{1}^{2}\right)\right) \\
= & -2\left(u_{1} u_{2}+\left(u_{-1} u_{1}-u_{1} u_{2}+2 u_{0} u_{1}\right)+\left(u_{-1} u_{-2}-u_{-1} u_{1}+u_{-1}^{2}-2 u_{0} u_{1}\right)\right. \\
& \left.-\left(u_{-2} u_{-1}+u_{-1}^{2}\right)\right) \\
= & 0 .
\end{aligned}
$$

This density is non-trivial since $\mathrm{E}(\rho)=2\left(u_{-1}+u_{0}+u_{1}\right) \neq 0$. One can quickly verify that $J=-2\left(u_{-1} u_{0} u_{1}+u_{0}^{2} u_{1}\right)$.

Obviously, $\rho=2 u_{0} u_{1}+u_{1}^{2}=2 u_{0} u_{1}+u_{0}^{2}+\left(u_{1}^{2}-u_{0}^{2}\right)=2 u_{0} u_{1}+u_{0}^{2}+\Delta\left(u_{0}^{2}\right)$. Hence, $\rho=2 u_{0} u_{1}+u_{0}^{2}$ and $\tilde{\rho}=2 u_{0} u_{1}+u_{1}^{2}$ are equivalent densities.

The computation of the action of the Euler operator quickly become tedious. However the Euler operator can be easily implemented in a computer algebra system like Maple, Mathematica or muPAD (Göktaş \& Hereman 1997, Hereman \& Göktaş 1999).

The computation of densities involves solving the integrability condition (2.15). This is a rather unusual "PDE" for $\rho$ since it involves derivatives of both $\rho$ and shifts of $\rho$. For example, if we assume that $\rho$ depends (among others) on the variable $u_{k}$ then $\mathrm{D}_{t} \rho$ will have a term

$$
\left(\frac{\partial}{\partial u_{k}} \rho\left(\ldots, u_{k}, \ldots\right)\right) \mathrm{D}^{k} f\left(u_{-l}, \ldots u_{m}\right) .
$$

This term will then give rise to terms of the form (the list is not exhaustive)

$$
\mathrm{D}^{-r}\left(\frac{\partial}{\partial u_{r}}\left(\left(\frac{\partial}{\partial u_{k}} \rho\left(\ldots, u_{k}, \ldots\right)\right) f\left(u_{k-l}, \ldots u_{k+m}\right)\right)\right),
$$

where $r$ ranges from $k-l$ to $k+m$ in the integrability condition (2.15). For example, with $r=k$, we get the term

$$
\frac{\partial}{\partial u_{0}}\left(\left(\frac{\partial}{\partial u_{0}} \rho\left(\ldots, u_{0}, \ldots\right)\right) f\left(u_{-l}, \ldots u_{m}\right)\right),
$$

whereas, with $r=k+1$, we get the term

$$
\frac{\partial}{\partial u_{0}}\left(\left(\frac{\partial}{\partial u_{-1}} \rho\left(\ldots, u_{-1}, \ldots\right)\right) f\left(u_{1-l}, \ldots u_{m+1}\right)\right) .
$$

These two terms have derivatives of $\rho$ evaluated with different arguments. It is then, perhaps, surprising that this equation is amenable to analysis. At this stage, it is best to proceed with an explicit example.

\section{Computation of Conservation Laws for DDEs. An Example}

Consider the modified Volterra (mV) lattice (Adler et al. 1999)

$$
\frac{d v_{n}}{d t}=v_{n}^{2}\left(v_{n+1}-v_{n-1}\right),
$$


or, equivalently,

$$
\frac{d v_{0}}{d t}=v_{0}^{2}\left(v_{1}-v_{-1}\right)
$$

To keep matters as clear as possible, we search for densities that depend only on the variables $v_{0}, v_{1}, v_{2}$; viz.

$$
\rho=\rho\left(v_{0}, v_{1}, v_{2}\right) .
$$

Attempting to find densities of greater generality would lead to messier, less illuminating calculations.

Upon differentiation

$$
\mathrm{D}_{t} \rho=v_{0}^{2}\left(v_{1}-v_{-1}\right) \frac{\partial}{\partial v_{0}} \rho+v_{1}^{2}\left(v_{2}-v_{0}\right) \frac{\partial}{\partial v_{1}} \rho+v_{2}^{2}\left(v_{3}-v_{1}\right) \frac{\partial}{\partial v_{2}} \rho .
$$

The integrability condition (2.15) reads

$$
\begin{aligned}
\mathrm{E}\left(\mathrm{D}_{t} \rho\right)= & v_{-1}^{2}\left(\frac{\partial}{\partial v_{-1}} \rho\left(v_{-3}, v_{-2}, v_{-1}\right)+\frac{\partial}{\partial v_{-1}} \rho\left(v_{-2}, v_{-1}, v_{0}\right)+\frac{\partial}{\partial v_{-1}} \rho\left(v_{-1}, v_{0}, v_{1}\right)\right) \\
& -v_{1}^{2}\left(\frac{\partial}{\partial v_{1}} \rho\left(v_{-1}, v_{0}, v_{1}\right)+\frac{\partial}{\partial v_{1}} \rho\left(v_{0}, v_{1}, v_{2}\right)+\frac{\partial}{\partial v_{1}} \rho\left(v_{1}, v_{2}, v_{3}\right)\right) \\
& +2 v_{0}\left(v_{1}-v_{-1}\right)\left(\frac{\partial}{\partial v_{0}} \rho\left(v_{-2}, v_{-1}, v_{0}\right)+\frac{\partial}{\partial v_{0}} \rho\left(v_{-1}, v_{0}, v_{1}\right)+\frac{\partial}{\partial v_{0}} \rho\left(v_{0}, v_{1}, v_{2}\right)\right) \\
& +v_{-1}^{2}\left(v_{0}-v_{-2}\right)\left(\frac{\partial^{2}}{\partial v_{-1} \partial v_{0}} \rho\left(v_{-2}, v_{-1}, v_{0}\right)+\frac{\partial^{2}}{\partial v_{-1} \partial v_{0}} \rho\left(v_{-1}, v_{0}, v_{1}\right)\right) \\
& +v_{0}^{2}\left(v_{1}-v_{-1}\right)\left(\frac{\partial^{2}}{\partial v_{0}^{2}} \rho\left(v_{-2}, v_{-1}, v_{0}\right)+\frac{\partial^{2}}{\partial v_{0}^{2}} \rho\left(v_{-1}, v_{0}, v_{1}\right)+\frac{\partial^{2}}{\partial v_{0}^{2}} \rho\left(v_{0}, v_{1}, v_{2}\right)\right) \\
& +v_{1}^{2}\left(v_{2}-v_{0}\right)\left(\frac{\partial^{2}}{\partial v_{0} \partial v_{1}} \rho\left(v_{-1}, v_{0}, v_{1}\right)+\frac{\partial^{2}}{\partial v_{0} \partial v_{1}} \rho\left(v_{0}, v_{1}, v_{2}\right)\right) \\
& +v_{-2}^{2}\left(v_{-1}-v_{-3}\right) \frac{\partial^{2}}{\partial v_{-2} \partial v_{0}} \rho\left(v_{-2}, v_{-1}, v_{0}\right)+v_{2}^{2}\left(v_{3}-v_{1}\right) \frac{\partial^{2}}{\partial v_{0} \partial v_{2}} \rho\left(v_{0}, v_{1}, v_{2}\right) \\
= & 0 .
\end{aligned}
$$

This illustrates the unusual nature of the integrability condition for $\rho$. It is a PDE for $\rho$. However the unknown function $\rho$ occurs with different arguments. Indeed, there are terms that involve derivatives of $\rho\left(v_{0}, v_{1}, v_{2}\right)$; other terms involve derivatives of $\mathrm{D}^{-1} \rho=\rho\left(v_{-1}, v_{0}, v_{1}\right)$, for example. Nonetheless, we can decompose the equation by differentiating with respect to various $v_{k}$ and separation of variables.

The highest shifted variable that occurs is $v_{3}$. Therefore, we differentiate with respect to $v_{3}$,

$$
v_{2}^{2} \frac{\partial^{2}}{\partial v_{0} \partial v_{2}} \rho\left(v_{0}, v_{1}, v_{2}\right)-v_{1}^{2} \frac{\partial^{2}}{\partial v_{1} \partial v_{3}} \rho\left(v_{1}, v_{2}, v_{3}\right)=0 .
$$

A further differentiation with respect to $v_{0}$ yields

$$
v_{2}^{2} \frac{\partial^{3}}{\partial v_{0}^{2} \partial v_{2}} \rho\left(v_{0}, v_{1}, v_{2}\right)=0
$$

Article submitted to Royal Society 
So,

$$
\begin{aligned}
\rho\left(v_{0}, v_{1}, v_{2}\right) & =\rho^{(1)}\left(v_{0}, v_{1}\right)-\rho^{(2)}\left(v_{0}, v_{1}\right)+\rho^{(2)}\left(v_{1}, v_{2}\right)+\rho^{(3)}\left(v_{1}, v_{2}\right) v_{0} \\
& =\rho^{(1)}\left(v_{0}, v_{1}\right)+\Delta \rho^{(2)}\left(v_{0}, v_{1}\right)+\rho^{(3)}\left(v_{1}, v_{2}\right) v_{0}
\end{aligned}
$$

for some unknown functions $\rho^{(1)}, \rho^{(2)}$ and $\rho^{(3)}$. The term $\Delta \rho^{(2)}\left(v_{0}, v_{1}\right)$ leads to a trivial density and can be ignored. Thus

$$
\rho=\rho^{(1)}\left(v_{0}, v_{1}\right)+\rho^{(3)}\left(v_{1}, v_{2}\right) v_{0} .
$$

The integrability condition (3.4) is now

$$
v_{2}^{2} \frac{\partial}{\partial v_{2}} \rho^{(3)}\left(v_{1}, v_{2}\right)-v_{1}^{2} \frac{\partial}{\partial v_{3}} \rho^{(3)}\left(v_{2}, v_{3}\right)=0 .
$$

A subsequent differentiation with respect to $v_{3}$ gives

$$
-v_{1}^{2} \frac{\partial^{2}}{\partial v_{3}^{2}} \rho^{(3)}\left(v_{2}, v_{3}\right)=0 .
$$

Consequently,

$$
\rho^{(3)}\left(v_{2}, v_{3}\right)=\rho^{(4)}\left(v_{2}\right)+\rho^{(5)}\left(v_{2}\right) v_{3} .
$$

Equation (3.4) has become

$$
v_{2}^{2} \rho^{(5)}\left(v_{1}\right)-v_{1}^{2} \rho^{(5)}\left(v_{2}\right)=0 .
$$

Thus,

$$
\frac{1}{v_{2}^{2}} \rho^{(5)}\left(v_{2}\right)=\frac{1}{v_{1}^{2}} \rho^{(5)}\left(v_{1}\right),
$$

from which we get

$$
\rho^{(5)}\left(v_{2}\right)=c^{(1)} v_{2}^{2}
$$

for some constant $c^{(1)}$

The density now has the form

$$
\rho=\rho^{(1)}\left(v_{0}, v_{1}\right)+\left(\rho^{(4)}\left(v_{1}\right)+c^{(1)} v_{1}^{2} v_{2}\right) v_{0} .
$$

The term $\rho^{(4)}\left(v_{1}\right) v_{0}$ can be absorbed into the term $\rho^{(1)}\left(v_{0}, v_{1}\right)$. Thus

$$
\rho\left(v_{0}, v_{1}, v_{2}\right)=\rho^{(1)}\left(v_{0}, v_{1}\right)+c^{(1)} v_{0} v_{1}^{2} v_{2} .
$$

At this stage we have reduced the problem from one unknown function of three variables to one unknown function of two variables and a constant.

Differentiating equation (3.3) with respect to $v_{2}$ twice yields

$$
v_{1}^{2}\left(2 c^{(1)} v_{1}-\frac{\partial^{3}}{\partial v_{2}^{2} \partial v_{1}} \rho^{(1)}\left(v_{1}, v_{2}\right)\right)=0,
$$

which readily integrates into

$$
\rho^{(1)}\left(v_{1}, v_{2}\right)=\frac{1}{2} c^{(1)} v_{1}^{2} v_{2}^{2}+\rho^{(6)}\left(v_{2}\right)+\rho^{(7)}\left(v_{1}\right)+\rho^{(8)}\left(v_{1}\right) v_{2} .
$$


Again we can write

$$
\rho^{(6)}\left(v_{2}\right)=\Delta \rho^{(6)}\left(v_{1}\right)+\rho^{(6)}\left(v_{1}\right)
$$

and absorb the term $\rho^{(6)}\left(v_{1}\right)$ into $\rho^{(7)}\left(v_{1}\right)$. Thus,

$$
\rho=\frac{1}{2} c^{(1)} v_{0}^{2} v_{1}^{2}+\rho^{(7)}\left(v_{0}\right)+\rho^{(8)}\left(v_{0}\right) v_{1}+c^{(1)} v_{0} v_{1}^{2} v_{2} .
$$

The $v_{2}$ derivative (3.3) is now

$$
v_{1}^{2}\left(\frac{d}{d v_{1}} \rho^{(8)}\left(v_{1}\right)-\frac{d}{d v_{0}} \rho^{(8)}\left(v_{0}\right)\right)=0,
$$

which yields

$$
\rho^{(8)}\left(v_{0}\right)=c^{(2)} v_{0}+c^{(3)}
$$

with constants $c^{(2)}$ and $c^{(3)}$. As before, the term $c^{(3)} v_{1}$ can be written as $\Delta c^{(3)} v_{0}+$ $c^{(3)} v_{0}$. Thus the density simplifies into

$$
\rho=\frac{1}{2} c^{(1)} v_{0}^{2} v_{1}^{2}+\rho^{(7)}\left(v_{0}\right)+c^{(2)} v_{0} v_{1}+c^{(1)} v_{0} v_{1}^{2} v_{2} .
$$

Eq. (3.3) is now reduced to

$$
\begin{aligned}
v_{0}^{2}\left(v_{1}-v_{-1}\right) \frac{d^{2}}{d v_{0}^{2}} \rho^{(7)}\left(v_{0}\right) & +2 v_{0}\left(v_{1}-v_{-1}\right) \frac{d}{d v_{0}} \rho^{(7)}\left(v_{0}\right) \\
+ & v_{-1}^{2} \frac{d}{d v_{-1}} \rho^{(7)}\left(v_{-1}\right)-v_{1}^{2} \frac{d}{d v_{1}} \rho^{(7)}\left(v_{1}\right)=0
\end{aligned}
$$

Differentiating twice with respect to $v_{1}$ yields the ordinary differential equation

$$
\frac{d^{2}}{d v_{1}^{2}}\left(v_{1}^{2} \frac{d}{d v_{1}} \rho^{(7)}\left(v_{1}\right)\right)=0 .
$$

Integration gives

$$
\rho^{(7)}\left(v_{1}\right)=c^{(4)}+c^{(5)} \frac{1}{v_{1}}+c^{(6)} \log v_{1}
$$

for some constants $c^{(4)}, c^{(5)}$ and $c^{(6)}$. The term $c^{(4)}$ gives a trivial density. Hence,

$$
\rho=\frac{1}{2} c^{(1)}\left(v_{0}^{2} v_{1}^{2}+2 v_{0} v_{1}^{2} v_{2}\right)+c^{(2)} v_{0} v_{1}+c^{(5)} \frac{1}{v_{0}}+c^{(6)} \log v_{0} .
$$

Eq. (3.3) is now satisfied. Hence, for (3.2) we have four independent, non-trivial densities that depend on, at most, three shifts. Namely,

$$
\begin{aligned}
\rho_{(1)} & =\frac{1}{v_{0}} \\
\rho_{(2)} & =\log v_{0} \\
\rho_{(3)} & =v_{0} v_{1} \\
\rho_{(4)} & =v_{0} v_{1}^{2}\left(\frac{1}{2} v_{0}+v_{2}\right),
\end{aligned}
$$

in agreement with the results in Adler et al. 1999. 
The associated fluxes are computed via $J_{(k)}=-\Delta^{-1}\left(\mathrm{D}_{t} \rho_{(k)}\right)$. They are

$$
\begin{aligned}
& J_{(1)}=v_{0}+v_{-1} \\
& J_{(2)}=-v_{-1} v_{0} \\
& J_{(3)}=-v_{-1} v_{0}^{2} v_{1} \\
& J_{(4)}=-v_{-1} v_{0}^{2} v_{1}^{2}\left(v_{0}+v_{2}\right) .
\end{aligned}
$$

This strategy of pealing off the terms that involve the highest (or lowest) shifted variables appears to work in many cases.

\section{Computing Fluxes and a Second Method to Compute Densities}

Densities $\rho$ and fluxes $J$ are related by (2.3). In principle, to compute $J=-\Delta^{-1}\left(\mathrm{D}_{t} \rho\right)$, one needs to invert the operator $\Delta=\mathrm{D}-\mathrm{I}$.

Working with the formal inverse,

$$
\Delta^{-1}=\mathrm{D}^{-1}+\mathrm{D}^{-2}+\mathrm{D}^{-3}+\ldots,
$$

is impractical, perhaps impossible.

We therefore present a simple algorithm which circumvents the above infinite formal series. Furthermore, starting from a candidate density the algorithm allows one to compute constraints for the density. For example, if the candidate has undetermined constant coefficients, one can derive the linear system for these unknown coefficients. If the candidate density is an unknown function, one can determine conditions for that function.

The idea is to split expressions into a total derivative term plus a term involving lower order shifts. Using

$$
\mathrm{I}=(\mathrm{D}-\mathrm{I}+\mathrm{I}) \mathrm{D}^{-1}=\Delta \mathrm{D}^{-1}+\mathrm{D}^{-1},
$$

any expression $T$ can be split as follows,

$$
T=\Delta \mathrm{D}^{-1} T+\mathrm{D}^{-1} T .
$$

The first term will contribute to the flux whilst the second term has a strictly lower shift than the original expression. These decompositions are applied to terms that do not involve the lowest-order shifted variables. Once all terms are "reduced" in this manner, any left-over terms (all of which involve the lowest-order shifted variable) are the obstruction. In case of a density the obstruction will be zero, otherwise the obstruction yields the constraints for the undetermined coefficients or unknown function.

So, for any density $\rho$ we compute $\mathrm{D}_{t} \rho$ and split it into

$$
\mathrm{D}_{t} \rho=A^{(0)}+A^{(1)},
$$

where $A^{(0)}$ is the part that does not depend on the lowest shifted variable $u_{p}$ that appears in $\mathrm{D}_{t} \rho$. Using (4.2), we write

$$
\mathrm{D}_{t} \rho=\Delta \mathrm{D}^{-1} A^{(0)}+\mathrm{D}^{-1} A^{(0)}+A^{(1)} .
$$


Now, if $\mathrm{D}^{-1} A^{(0)}+A^{(1)}=0$ we are finished and $J=-\mathrm{D}^{-1} A^{(0)}$. If $\mathrm{D}^{-1} A^{(0)}+A^{(1)} \neq 0$ we again split this term,

$$
\mathrm{D}^{-1} A^{(0)}+A^{(1)}=A^{(2)}+A^{(3)}
$$

where $A^{(2)}$ does not depend on $u_{p}$. Applying (4.2) to $A^{(2)}$ we get

$$
\mathrm{D}_{t} \rho=\Delta \mathrm{D}^{-1} A^{(0)}+\Delta \mathrm{D}^{-1} A^{(2)}+\mathrm{D}^{-1} A^{(2)}+A^{(3)} .
$$

If $\mathrm{D}^{-1} A^{(2)}+A^{(3)}=0$ then the flux is $J=-\mathrm{D}^{-1}\left(A^{(0)}+A^{(2)}\right)$. If not, we repeat the process. This procedure must eventually terminate since $\rho$ is a density.

We illustrate this algorithm for the $\mathrm{mV}$ lattice (3.2) with density $\rho=\log v_{0}$. Now,

$$
\mathrm{D}_{t} \log v_{0}=\frac{v_{0}^{2}\left(v_{1}-v_{-1}\right)}{v_{0}}=v_{0}\left(v_{1}-v_{-1}\right)
$$

So, we have $A^{(0)}=v_{0} v_{1}$ and $A^{(1)}=-v_{-1} v_{0}$. Thus

$$
\mathrm{D}_{t} \log v_{0}=\Delta \mathrm{D}^{-1} v_{0} v_{1}+\mathrm{D}^{-1} v_{0} v_{1}-v_{-1} v_{0}=\Delta v_{-1} v_{0}
$$

giving $J=-v_{-1} v_{0}$. In this simple case the algorithm terminated after one step.

More importantly, the above algorithm can be applied to the problem of computing densities. Without loss of generality, we can assume that $\rho=\rho\left(u_{0}, \ldots, u_{q}\right)$. For (2.2), we have

$$
\begin{aligned}
\mathrm{D}_{t} \rho & =\sum_{k=0}^{q} \frac{\partial \rho}{\partial u_{k}} \mathrm{D}^{k} f\left(u_{-l}, \ldots, u_{m}\right) \\
& =\frac{\partial \rho}{\partial u_{0}} f\left(u_{-l}, \ldots, u_{m}\right)+\sum_{k=1}^{q} \frac{\partial \rho}{\partial u_{k}} \mathrm{D}^{k} f\left(u_{-l}, \ldots, u_{m}\right) .
\end{aligned}
$$

Applying (4.2) to the second term we obtain

$$
\begin{aligned}
\mathrm{D}_{t} \rho & =\frac{\partial \rho}{\partial u_{0}} f+\left(\Delta \mathrm{D}^{-1}+\mathrm{D}^{-1}\right)\left(\sum_{k=1}^{q} \frac{\partial \rho}{\partial u_{k}} \mathrm{D}^{k} f\right) \\
& =\frac{\partial \rho}{\partial u_{0}} f+(\Delta+\mathrm{I})\left(\sum_{k=1}^{q} \frac{\partial \mathrm{D}^{-1} \rho}{\partial u_{k-1}} \mathrm{D}^{k-1} f\right) \\
& =\frac{\partial \rho}{\partial u_{0}} f+\Delta \sum_{k=0}^{q-1} \frac{\partial \mathrm{D}^{-1} \rho}{\partial u_{k}} \mathrm{D}^{k} f+\sum_{k=0}^{q-1} \frac{\partial \mathrm{D}^{-1} \rho}{\partial u_{k}} \mathrm{D}^{k} f \\
& =\left(\frac{\partial}{\partial u_{0}}\left(\rho+\mathrm{D}^{-1} \rho\right)\right) f+\Delta \sum_{k=0}^{q-1} \frac{\partial \mathrm{D}^{-1} \rho}{\partial u_{k}} \mathrm{D}^{k} f+\sum_{k=1}^{q-1} \frac{\partial \mathrm{D}^{-1} \rho}{\partial u_{k}} \mathrm{D}^{k} f
\end{aligned}
$$

Article submitted to Royal Society 
with $f=f\left(u_{-l}, \ldots, u_{m}\right)$. Next, we repeat this procedure by applying (4.2) to the last term. After a further $q-2$ applications, we get

$$
\begin{aligned}
\mathrm{D}_{t} \rho= & \left(\frac{\partial}{\partial u_{0}}\left(\rho+\mathrm{D}^{-1} \rho+\mathrm{D}^{-2} \rho\right)\right) f+\Delta\left(\sum_{k=0}^{q-1} \frac{\partial \mathrm{D}^{-1} \rho}{\partial u_{k}} \mathrm{D}^{k} f+\sum_{k=0}^{q-2} \frac{\partial \mathrm{D}^{-2} \rho}{\partial u_{k}} \mathrm{D}^{k} f\right) \\
= & \quad+\sum_{k=1}^{q-2} \frac{\partial \mathrm{D}^{-2} \rho}{\partial u_{k}} \mathrm{D}^{k} f \\
= & \left(\frac{\partial}{\partial u_{0}} \sum_{j=0}^{q} \mathrm{D}^{-j} \rho\right) f+\Delta\left(\sum_{j=1}^{q} \sum_{k=0}^{q-j} \frac{\partial \mathrm{D}^{-j} \rho}{\partial u_{k}} \mathrm{D}^{k} f\right) \\
= & \mathrm{E}(\rho) f+\Delta\left(\sum_{j=1}^{q} \sum_{k=0}^{q-j} \frac{\partial \mathrm{D}^{-j} \rho}{\partial u_{k}} \mathrm{D}^{k} f\right)
\end{aligned}
$$

by $(2.11)$.

If $\mathrm{E}(\rho) f=0$ then $\rho$ is a trivial density. For $\rho$ to be a non-trivial density, we require

$$
\mathrm{E}(\rho) f=\Delta h
$$

for some $h$ with $h \neq 0$. In this case the associated flux is

$$
J=-h-\sum_{j=1}^{q} \sum_{k=0}^{q-j} \frac{\partial \mathrm{D}^{-j} \rho}{\partial u_{k}} \mathrm{D}^{k} f .
$$

One could apply the discrete Euler operator to $\mathrm{E}(\rho) f$ to determine conditions such that (4.3) holds. Alternatively, one could repeat the above strategy by splitting this expression into a part, $A^{(0)}$, that does not depend on the lowest shifted variable and the remaining terms. One then applies (4.2) repeatedly to $A^{(0)}$ (removing the total difference terms generated by (4.2) from $A^{(0)}$ ) until one obtains a term that involves both the lowest and highest shifted variables. By noting that, from (2.13), a necessary condition that an $g=g\left(u_{p}, \ldots, u_{q}\right)$ is a total difference is

$$
\frac{\partial^{2}}{\partial u_{p} \partial u_{q}} g=0
$$

one then obtains conditions for (4.3) to hold. This procedure must be repeated until all the terms in $\mathrm{E}(\rho) f$ have been recast as a total difference.

We now illustrate this approach by recomputing low order densities for the $\mathrm{mV}$ lattice.

\section{The Modified Volterra Lattice Revisited}

To keep the exposition simple, we search for densities of the form

$$
\rho=\rho\left(v_{0}, v_{1}, v_{2}\right)
$$

for the $\mathrm{mV}$ lattice (3.2), where $f=v_{0}^{2}\left(v_{1}-v_{-1}\right)$. The construction of densities involving a greater spread of dynamic variables could be attempted with a computer algebra package. 
We write

$$
\mathrm{D}_{t} \rho=\sigma+\Delta K
$$

with

$$
\begin{aligned}
\sigma & =\mathrm{E}(\rho) f \\
& =v_{0}^{2}\left(v_{1}-v_{-1}\right)\left(\frac{\partial}{\partial v_{0}} \rho\left(v_{0}, v_{1}, v_{2}\right)+\frac{\partial}{\partial v_{0}} \rho\left(v_{-1}, v_{0}, v_{1}\right)+\frac{\partial}{\partial v_{0}} \rho\left(v_{-2}, v_{-1}, v_{0}\right)\right)
\end{aligned}
$$

and

$$
\begin{aligned}
K= & \sum_{j=1}^{q} \sum_{k=0}^{q-j} \frac{\partial \mathrm{D}^{-j} \rho}{\partial u_{k}} \mathrm{D}^{k} f \\
= & v_{0}^{2}\left(v_{1}-v_{-1}\right) \frac{\partial}{\partial v_{0}} \rho\left(v_{-1}, v_{0}, v_{1}\right)+v_{1}^{2}\left(v_{2}-v_{0}\right) \frac{\partial}{\partial v_{1}} \rho\left(v_{-1}, v_{0}, v_{1}\right) \\
& \quad+v_{0}^{2}\left(v_{1}-v_{-1}\right) \frac{\partial}{\partial v_{0}} \rho\left(v_{-2}, v_{-1}, v_{0}\right)
\end{aligned}
$$

The application of (4.4) directly to $\sigma$ yields nothing since no term depends on both the lowest shifted variable $\left(v_{-2}\right)$ and the highest shifted variable $\left(v_{2}\right)$. We therefore split $\sigma$ into a term which depends on $v_{-2}$ and a term that does not. We then apply (4.2) to the latter term.

Thus,

$$
\begin{aligned}
\sigma= & v_{0}^{2}\left(v_{1}-v_{-1}\right) \frac{\partial}{\partial v_{0}} \rho\left(v_{-2}, v_{-1}, v_{0}\right) \\
& +(\Delta+\mathrm{I}) \mathrm{D}^{-1}\left(v_{0}^{2}\left(v_{1}-v_{-1}\right)\left(\frac{\partial}{\partial v_{0}} \rho\left(v_{0}, v_{1}, v_{2}\right)+\frac{\partial}{\partial v_{0}} \rho\left(v_{-1}, v_{0}, v_{1}\right)\right)\right) \\
= & v_{0}^{2}\left(v_{1}-v_{-1}\right) \frac{\partial}{\partial v_{0}} \rho\left(v_{-2}, v_{-1}, v_{0}\right) \\
& +(\Delta+\mathrm{I})\left(v_{-1}^{2}\left(v_{0}-v_{-2}\right)\left(\frac{\partial}{\partial v_{-1}} \rho\left(v_{-1}, v_{0}, v_{1}\right)+\frac{\partial}{\partial v_{-1}} \rho\left(v_{-2}, v_{-1}, v_{0}\right)\right)\right) .
\end{aligned}
$$

Next, we update $\sigma$ and $K$ by removing the total difference term from $\sigma$ and adding it to $K$. Thus,

$$
\begin{aligned}
\sigma=v_{0}^{2}\left(v_{1}\right. & \left.-v_{-1}\right) \frac{\partial}{\partial v_{0}} \rho\left(v_{-2}, v_{-1}, v_{0}\right) \\
& +v_{-1}^{2}\left(v_{0}-v_{-2}\right)\left(\frac{\partial}{\partial v_{-1}} \rho\left(v_{-1}, v_{0}, v_{1}\right)+\frac{\partial}{\partial v_{-1}} \rho\left(v_{-2}, v_{-1}, v_{0}\right)\right),
\end{aligned}
$$

and

$$
\begin{aligned}
K=v_{0}^{2}\left(v_{1}\right. & \left.-v_{-1}\right)\left(\frac{\partial}{\partial v_{0}} \rho\left(v_{-1}, v_{0}, v_{1}\right)+\frac{\partial}{\partial v_{0}} \rho\left(v_{-2}, v_{-1}, v_{0}\right)\right) \\
& +v_{1}^{2}\left(v_{2}-v_{0}\right) \frac{\partial}{\partial v_{1}} \rho\left(v_{-1}, v_{0}, v_{1}\right) \\
& +v_{-1}^{2}\left(v_{0}-v_{-2}\right)\left(\frac{\partial}{\partial v_{-1}} \rho\left(v_{-1}, v_{0}, v_{1}\right)+\frac{\partial}{\partial v_{-1}} \rho\left(v_{-2}, v_{-1}, v_{0}\right)\right) .
\end{aligned}
$$

Article submitted to Royal Society 
Now we are ready to apply (4.4) to $\sigma$. Thus,

$$
\frac{\partial^{2}}{\partial v_{-2} \partial v_{1}} \sigma=v_{0}^{2} \frac{\partial^{2}}{\partial v_{-2} \partial v_{0}} \rho\left(v_{-2}, v_{-1}, v_{0}\right)-v_{-1}^{2} \frac{\partial^{2}}{\partial v_{-1} \partial v_{1}} \rho\left(v_{-1}, v_{0}, v_{1}\right)=0 .
$$

Note that we get (3.4) of our first derivation by applying to up-shifts to the above equation. Therefore, the solution is given by (3.5):

$$
\rho=\rho^{(1)}\left(v_{0}, v_{1}\right)+c^{(1)} v_{0} v_{1}^{2} v_{2} .
$$

This procedure is repeated until $\sigma$ is reduced to 0 . When this happens $-K$ will be the associated flux. Hence,

$$
\begin{aligned}
\sigma= & c^{(1)} v_{-2} v_{-1}{ }^{3} v_{0}\left(v_{0}-2 v_{-2}\right)-v_{-2} v_{-1}^{2} \frac{\partial}{\partial v_{-1}} \rho^{(1)}\left(v_{-1}, v_{0}\right) \\
& +v_{-1}^{2}\left(v_{0}-v_{-2}\right) \frac{\partial}{\partial v_{-1}} \rho^{(1)}\left(v_{-2}, v_{-1}\right)+c^{(1)} v_{-1}^{2} v_{0}^{3} v_{1}+v_{-1}^{2} v_{0} \frac{\partial}{\partial v_{-1}} \rho^{(1)}\left(v_{-1}, v_{0}\right) \\
= & c^{(1)} v_{-2} v_{-1}{ }^{3} v_{0}\left(v_{0}-2 v_{-2}\right)-v_{-2} v_{-1}{ }^{2} \frac{\partial}{\partial v_{-1}} \rho^{(1)}\left(v_{-1}, v_{0}\right) \\
& +v_{-1}^{2}\left(v_{0}-v_{-2}\right) \frac{\partial}{\partial v_{-1}} \rho^{(1)}\left(v_{-2}, v_{-1}\right) \\
& +(\Delta+\mathrm{I})\left(c^{(1)} v_{-2}^{2} v_{-1}^{3} v_{0}+v_{-2}^{2} v_{-1} \frac{\partial}{\partial v_{-2}} \rho^{(1)}\left(v_{-2}, v_{-1}\right)\right)
\end{aligned}
$$

by (4.2). As before, we update $\sigma$ by moving the total derivative term into $K$.

Therefore,

$$
\begin{aligned}
\sigma=c^{(1)} v_{-2} v_{-1}{ }^{3} v_{0}\left(v_{0}-v_{-2}\right)-v_{-2} v_{-1}{ }^{2} \frac{\partial}{\partial v_{-1}} \rho^{(1)}\left(v_{-1}, v_{0}\right) \\
\quad+v_{-1}^{2}\left(v_{0}-v_{-2}\right) \frac{\partial}{\partial v_{-1}} \rho^{(1)}\left(v_{-2}, v_{-1}\right)+v_{-2}^{2} v_{-1} \frac{\partial}{\partial v_{-2}} \rho^{(1)}\left(v_{-2}, v_{-1}\right),
\end{aligned}
$$

and

$$
\begin{array}{r}
K=c^{(1)} v_{-1} v_{0}\left(v_{-1} v_{0}^{2} v_{1}-v_{0} v_{1}^{2} v_{2}-v_{-2} v_{-1}^{2} v_{0}-v_{0}^{2} v_{1}^{2}+3 v_{-2}^{2} v_{-1}^{2}\right) \\
+v_{0}^{2}\left(v_{-1}-v_{1}\right) \frac{\partial}{\partial v_{0}} \rho^{(1)}\left(v_{-1}, v_{0}\right)+v_{-2}^{2} v_{-1} \frac{\partial}{\partial v_{-2}} \rho^{(1)}\left(v_{-2}, v_{-1}\right) \\
+v_{-1}^{2}\left(v_{-2}-v_{0}\right)\left(\frac{\partial}{\partial v_{-1}} \rho^{(1)}\left(v_{-1}, v_{0}\right)+\frac{\partial}{\partial v_{-1}} \rho^{(1)}\left(v_{-2}, v_{-1}\right)\right) .
\end{array}
$$

Applying (4.4) to $\sigma$ yields

$$
\begin{aligned}
\frac{\partial^{2} \sigma}{\partial v_{-2} \partial v_{0}} & =v_{-1}^{2}\left(2 c^{(1)} v_{-1}\left(v_{0}-v_{-2}\right)-\frac{\partial^{2}}{\partial v_{-1} \partial v_{0}} \rho^{(1)}\left(v_{-1}, v_{0}\right)+\frac{\partial^{2}}{\partial v_{-2} \partial v_{-1}} \rho^{(1)}\left(v_{-2}, v_{-1}\right)\right) \\
& =0 .
\end{aligned}
$$

This equation is a shifted version of the $v_{2}$ derivative of (3.3). In turn, its $v_{0}$ derivative is a shifted version of (3.6). The solution is thus given by (3.7) after an appropriate shift:

$$
\rho=\frac{1}{2} c^{(1)} v_{0}^{2} v_{1}^{2}+\rho^{(7)}\left(v_{0}\right)+c^{(2)} v_{0} v_{1}+c^{(1)} v_{0} v_{1}^{2} v_{2} .
$$


At this stage

$$
\sigma=v_{-2}^{2} v_{-1} \frac{d}{d v_{-2}} \rho^{(7)}\left(v_{-2}\right)-v_{2} v_{-1}^{2} \frac{d}{d v_{-1}} \rho^{(7)}\left(v_{-1}\right)
$$

and

$$
\begin{aligned}
K=v_{-2}{ }^{2} v_{-1} & \frac{d}{d v_{-2}} \rho^{(7)}\left(v_{-2}\right)-v_{-1}^{2} v_{-2} \frac{d}{d v_{-1}} \rho^{(7)}\left(v_{-1}\right) \\
& +v_{0}^{3} c^{(1)} v_{-1} v_{1}^{2}+v_{-1}^{2} v_{0} \frac{d}{d v_{-1}} \rho^{(7)}\left(v_{-1}\right)+c^{(1)} v_{-1} v_{0}^{2} v_{1}^{2} v_{2}+v_{0}{ }^{2} c^{(2)} v_{-1} v_{1} .
\end{aligned}
$$

One more application of (4.4) to $\sigma$ yields

$$
\frac{\partial^{2} \sigma}{\partial v_{-2} \partial v_{-1}}=\frac{d}{d v_{-2}}\left(v_{-2}^{2} \frac{d}{d v_{-2}} \rho^{(7)}\left(v_{-2}\right)\right)-\frac{d}{d v_{-1}}\left(v_{-1}^{2} \frac{d}{d v_{-1}} \rho^{(7)}\left(v_{-1}\right)\right)=0 .
$$

Differentiating this equation with respect to $v_{-2}$ yields a shifted version of $(3.8)$. Thus the solution is given by (3.10):

$$
\rho=\frac{1}{2} c^{(1)}\left(v_{0}^{2} v_{1}^{2}+2 v_{0} v_{1}^{2} v_{2}\right)+c^{(2)} v_{0} v_{1}+c^{(5)} \frac{1}{v_{0}}+c^{(6)} \log v_{0} .
$$

Consequently,

$$
\sigma=c^{(5)} v_{-2}-c^{(5)} v_{-1}=-c^{(5)} \Delta v_{-2},
$$

which can be absorbed into $K$ to yield $\sigma=0$. Formally we could apply (4.2) to $\sigma$ but in this case the result is obvious. Finally, the associated flux is

$$
J=-K=-c^{(1)} v_{-1} v_{0}^{2} v_{1}^{2}\left(v_{0}+v_{2}\right)-c^{(2)} v_{-1} v_{0}^{2} v_{1}+c^{(5)}\left(v_{0}+v_{-1}\right)-c^{(6)} v_{-1} v_{0} .
$$

Splitting the density $\rho$ and flux $J$ according to the independent constants $c^{(i)}$ we obtain the densities and fluxes listed at the end of Section 3.

\section{Miura Map}

In this section we investigate the connection between the $\mathrm{KvM}$ and $\mathrm{mV}$ lattices.

In contrast to the approach in Yamilov 1994, we construct an explicit homotopic deformation of the KvM lattice and restrict it to retrieve the Miura map which connects both lattices. Recursive use of the map allows one to find the conserved densities for the KvM lattice order by order.

Starting from the KvM lattice (2.6),

$$
\frac{d u_{0}}{d t}=u_{0}\left(u_{1}-u_{-1}\right)
$$

consider the non-local map

$$
u_{0}=v_{0}+\epsilon F\left(v_{-1}, v_{0}\right) \text {, }
$$

where we assume that $F$ is analytic at $\epsilon=0$ (for notational simplicity, we suppress the dependency on $\epsilon$ ) so that $u_{0}=v_{0}$ at $\epsilon=0$. Under this transformation, the KvM lattice is deformed into

$$
\begin{aligned}
& \frac{d v_{0}}{d t}+\epsilon\left(\left(\frac{\partial}{\partial v_{-1}} F\left(v_{-1}, v_{0}\right)\right) \frac{d v_{-1}}{d t}+\left(\frac{\partial}{\partial v_{0}} F\left(v_{-1}, v_{0}\right)\right) \frac{d v_{0}}{d t}\right)= \\
&\left(v_{0}+\epsilon F\left(v_{-1}, v_{0}\right)\right)\left(v_{1}+\epsilon F\left(v_{0}, v_{1}\right)-v_{-1}-\epsilon F\left(v_{-2}, v_{-1}\right)(5.2)\right.
\end{aligned}
$$


This equation involves the shifted variables $v_{-2}, v_{-1}, v_{0}$ and $v_{1}$ So, we obtain an implicit non-local lattice for $v_{0}$ given by

$$
\frac{d v_{0}}{d t}=g\left(v_{-1}, v_{0}, v_{1}\right)
$$

Note that there is no guarantee that an explicit function $g$ will exist. In other words, the KvM lattice may be mapped onto a lattice which is not of type (2.2). Again, for clarity, the $\epsilon$ dependency of $g$ is suppressed. Eq. (5.2) is regarded as an integrability condition on $F$ so that $v_{0}$ does indeed satisfy (5.3). Map (5.1) is viewed as deforming (2.6) which is recovered by setting the parameter $\epsilon$ to 0 .

The KvM lattice (2.6) has density $\rho=\log u_{0}$. We impose the additional condition on map (5.1) that it preserves this density. That is, for all $\epsilon$ in a neighborhood of $0, \log v_{0}$ should be a density for (5.3). The resulting integrability condition is

$$
\begin{aligned}
\mathrm{E}\left(\mathrm{D}_{t} \log v_{0}\right) & =\mathrm{E}\left(\frac{g\left(v_{-1}, v_{0}, v_{1}\right)}{v_{0}}\right) \\
& =-\frac{1}{v_{0}^{2}} g\left(v_{-1}, v_{0}, v_{1}\right)+\frac{1}{v_{0}} \frac{\partial}{\partial v_{0}} g\left(v_{-1}, v_{0}, v_{1}\right)+\frac{1}{v_{1}} \frac{\partial}{\partial v_{0}} g\left(v_{0}, v_{1}, v_{2}\right) \\
& \quad+\frac{1}{v_{-1}} \frac{\partial}{\partial v_{0}} g\left(v_{-2}, v_{-1}, v_{0}\right) \\
& =0 .
\end{aligned}
$$

Differentiating the above with respect to $v_{-2}$ yields

$$
\frac{\partial^{2}}{\partial v_{0} \partial v_{-2}} g\left(v_{-2}, v_{-1}, v_{0}\right)=0
$$

and so

$$
g\left(v_{-1}, v_{0}, v_{1}\right)=g^{(1)}\left(v_{-1}, v_{0}\right)+g^{(2)}\left(v_{0}, v_{1}\right) .
$$

The $v_{-1}$ derivative of (5.4) can be written in the form

$$
\frac{\partial^{2}}{\partial v_{-1} \partial v_{0}}\left(\frac{1}{v_{0}} g^{(1)}\left(v_{-1}, v_{0}\right)+\frac{1}{v_{-1}} g^{(2)}\left(v_{-1}, v_{0}\right)\right)=0
$$

Thus we obtain

$$
g^{(2)}\left(v_{-1}, v_{0}\right)=-\frac{v_{-1}}{v_{0}} g^{(1)}\left(v_{-1}, v_{0}\right)+g^{(3)}\left(v_{-1}\right)+v_{-1} g^{(4)}\left(v_{0}\right) .
$$

Eq. (5.4) now reads

$$
\frac{\partial}{\partial v_{0}}\left(\frac{1}{v_{0}} g^{(3)}\left(v_{0}\right)+g^{(4)}\left(v_{0}\right)\right)=0
$$

and so

$$
g^{(4)}\left(v_{0}\right)=-\frac{1}{v_{0}} g^{(3)}\left(v_{0}\right)+c,
$$

where $c$ is a constant. The integrability condition is now satisfied, and

$g\left(v_{-1}, v_{0}, v_{1}\right)=g^{(1)}\left(v_{-1}, v_{0}\right)-\frac{v_{0}}{v_{1}} g^{(1)}\left(v_{0}, v_{1}\right)+g^{(3)}\left(v_{0}\right)+v_{0}\left(-\frac{1}{v_{1}} g^{(3)}\left(v_{1}\right)+c\right)$. 
Absorbing $g^{(3)}\left(v_{0}\right)$ into $g^{(1)}\left(v_{-1}, v_{0}\right)$, the most general lattice of form (5.3) that has a density $\log v_{0}$ is

$$
\frac{d v_{0}}{d t}=G\left(v_{-1}, v_{0}\right)-\frac{v_{0}}{v_{1}} G\left(v_{0}, v_{1}\right)+c v_{0},
$$

where $G$ is an arbitrary function of its variables. Note that for the choice $c=0$ and

$$
G\left(v_{-1}, v_{0}\right)=-v_{-1} v_{0}-v_{0}^{2}
$$

(5.5) is the KvM lattice (2.6). For the special case $c=0$ and

$$
G\left(v_{-1}, v_{0}\right)=-v_{-1} v_{0}^{2}
$$

(5.5) reduces to the $\mathrm{mV}$ lattice $(3.2)$.

Continuing with arbitrary $G\left(v_{-1}, v_{0}\right)$, we now ask if there exists a deformation (5.1) of the KvM lattice (2.6) such that the deformed lattice has the form (5.5) for all $\epsilon$ in a neighborhood of 0 . Obviously, this will further restrict the admissible functions $F$ and $G$.

The necessary condition is obtained by substituting (5.5) into (5.2). Differentiating the resulting expression with respect to $v_{-2}$ yields

$\epsilon\left(\frac{\partial}{\partial v_{-1}} F\left(v_{-1}, v_{0}\right)\right) \frac{\partial}{\partial v_{-2}} G\left(v_{-2}, v_{-1}\right)=-\left(v_{0}+\epsilon F\left(v_{-1}, v_{0}\right)\right) \epsilon \frac{\partial}{\partial v_{-2}} F\left(v_{-2}, v_{-1}\right)$.

Assuming that the deformation (5.1) is genuinely non-local, consequently, that $F\left(v_{-1}, v_{0}\right)$ depends non-trivially on $v_{-1}$, we may rewrite this equation as

$$
\frac{\frac{\partial}{\partial v_{-2}} G\left(v_{-2}, v_{-1}\right)}{\frac{\partial}{\partial v_{-2}} F\left(v_{-2}, v_{-1}\right)}=-\frac{v_{0}+\epsilon F\left(v_{-1}, v_{0}\right)}{\frac{\partial}{\partial v_{-1}} F\left(v_{-1}, v_{0}\right)} \text {. }
$$

Since the left hand side is independent of $v_{0}$ and $F$ is analytic at $\epsilon=0$,

$$
F\left(v_{-1}, v_{0}\right)=v_{0} F^{(1)}\left(v_{-1}\right)
$$

with

$$
\frac{\frac{\partial}{\partial v_{-2}} G\left(v_{-2}, v_{-1}\right)}{\frac{d}{d v_{-2}} F^{(1)}\left(v_{-2}\right)}=-\frac{v_{-1}\left(1+\epsilon F^{(1)}\left(v_{-1}\right)\right)}{\frac{d}{d v_{-1}} F^{(1)}\left(v_{-1}\right)} .
$$

The left hand side is independent of $v_{-2}$. Thus,

$$
G\left(v_{-2}, v_{-1}\right)=-\frac{v_{-1}\left(1+\epsilon F^{(1)}\left(v_{-1}\right)\right)}{\frac{d}{d v_{-1}} F^{(1)}\left(v_{-1}\right)} F^{(1)}\left(v_{-2}\right)+G^{(1)}\left(v_{-1}\right) .
$$

Differentiating (5.2) with respect to both $v_{-1}$ and $v_{1}$ yields

$$
\begin{aligned}
& v_{1}^{2} F^{(1)}\left(v_{0}\right) \frac{d^{2} F^{(1)}\left(v_{1}\right)}{d v_{1}^{2}}\left(1+\epsilon F^{(1)}\left(v_{1}\right)\right) \\
& +\left(\frac{d F^{(1)}\left(v_{1}\right)}{d v_{1}}\right)^{2}\left(v_{1}^{2}+v_{1} \frac{d G^{(1)}\left(v_{1}\right)}{d v_{1}}-G^{(1)}\left(v_{1}\right)\right)=0 .
\end{aligned}
$$

Article submitted to Royal Society 
A further differentiation with respect to $v_{0}$ gives

$$
v_{1}^{2}\left(\frac{d F^{(1)}\left(v_{0}\right)}{d v_{0}}\right)\left(\frac{d^{2} F^{(1)}\left(v_{1}\right)}{d v_{1}^{2}}\right)\left(1+\epsilon F^{(1)}\left(v_{1}\right)\right)=0,
$$

and so

$$
F^{(1)}\left(v_{1}\right)=v_{1}+a
$$

for some constant $a$. The coefficient of $v_{1}$ can be taken 1 by rescaling $\epsilon$ if necessary. Differentiating (5.2) twice with respect to $v_{-1}$ yields

$$
\epsilon v_{0}\left(2+\frac{d^{2}}{d v_{-1}^{2}} G^{(1)}\left(v_{-1}\right)\right)=0
$$

and so

$$
G^{(1)}\left(v_{-1}\right)=-v_{-1}^{2}+b^{(1)} v_{-1}+b^{(2)}
$$

for some constants $b^{(1)}$ and $b^{(2)}$. Equation (5.2) now reads

$-\left(c+\epsilon a c+\epsilon b^{(2)}\right) v_{0} v_{1}-2 c \epsilon v_{-1} v_{0} v_{1}+b^{(2)}(1+a \epsilon)\left(v_{0}-v_{1}\right)+b^{(2)} \epsilon v_{-1} v_{0}=0$

Thus $c=0$ and $b^{(2)}=0$. Therefore the required deformation (5.1) is

$$
u_{0}=v_{0}+\epsilon\left(v_{-1}+a\right) v_{0}=(1+\epsilon a) v_{0}+\epsilon v_{1} v_{0},
$$

and the deformed lattice (5.5) is

$$
\frac{d v_{0}}{d t}=v_{0}\left(1+\epsilon a+\epsilon v_{0}\right)\left(v_{1}-v_{-1}\right) .
$$

For $a \neq 0,(5.7)$ is the $\mathrm{mV}$ lattice (3.2) when $\epsilon=-a^{-1}$. Thus the deformation (5.6) is a homotopy between the $\mathrm{KvM}$ and the $\mathrm{mV}$ lattices. For $a=0$ the $\mathrm{mV}$ lattice is obtained in the limit $\epsilon \rightarrow \infty$. For $a=-1$ and $\epsilon=1$, the upshifted version of (5.6) is a well-known nonlinear transformation (Adler et al. 1999, Levi \& Yamilov 1997),

$$
u_{0}=v_{0} v_{1}
$$

which maps solutions of (3.2) into solutions of (2.6).

Map (5.6) is the analogue of the famous Miura transformation connecting the $\mathrm{KdV}$ and modified KdV equations (Miura 1968). For other non-zero values of $\epsilon$, (5.7) is a combined $\mathrm{KvM}-\mathrm{mV}$ lattice.

Finally, we use the homotopy (5.6) to generate conserved densities of the KvM lattice. The strategy is similar to the one for the continuous case (Miura et al. 1968) where the Miura transformation was used to find low-order conserved densities for the KdV equation.

Since $\log v_{0}$ is a density for all the deformed equations (that is, independent of $\epsilon)$, we can compute the densities of the KvM lattice (2.6) by recursively substituting (5.6) into $\log v_{0}$ and expanding in powers of $\epsilon$. Each coefficient must therefore be a density of the KvM lattice. Conveniently, we set $a=0$. The deformation (5.6) can be recursively solved for $v_{0}$

$$
v_{0}=\frac{u_{0}}{1+\epsilon v_{-1}},
$$

Article submitted to Royal Society 
and so

$$
\rho=\log v_{0}=\log \left(\frac{u_{0}}{1+\epsilon v_{-1}}\right)=\log u_{0}+\mathrm{O}(\epsilon) .
$$

Not surprisingly, $\log u_{0}$ is a density of the KvM lattice. Repeating this procedure, we obtain

$$
\begin{aligned}
\rho & =\log \left(\frac{u_{0}}{1}+\frac{\epsilon u_{-1}}{1+\epsilon v_{-2}}\right) \\
& =\log u_{0}-u_{-1} \epsilon+\mathrm{O}\left(\epsilon^{2}\right)
\end{aligned}
$$

Therefore $u_{-1}$ (equivalently $u_{0}$ ) is also a density of the KvM lattice. Repeating this procedure once more,

$$
\begin{aligned}
\rho & =\log \left(u_{0}\left(1+\epsilon u_{-1}\left(1+\frac{\epsilon u_{-2}}{1+\epsilon v_{-3}}\right)^{-1}\right)^{-1}\right) \\
& =\log u_{0}-u_{-1} \epsilon+\frac{1}{2} u_{-1}\left(2 u_{-2}+u_{-1}\right) \epsilon^{2}+\mathrm{O}\left(\epsilon^{3}\right)
\end{aligned}
$$

After a shift, the next density of the KvM lattice is $u_{0}\left(2 u_{-1}+u_{0}\right)$.

Although cumbersome, higher-order densities of the KvM lattice could be obtained this way.

Conversely, apart from density $\frac{1}{v_{0}}$, the densities (3.11) can be obtained from the densities of the KvM lattice using (5.8).

\section{Conclusion}

The two methods discussed in this paper allow one to find conserved densities and fluxes of differential-difference equations.

Starting with a conserved density of a particular form, the first method relied on finding a closed-form solution of the integrability condition which follows from the application of the discrete Euler operator. For the example of the $\mathrm{mV}$ lattice, the solution was obtained by repeated differentiation and separation of variables. The more general the assumed form of the density, the more cumbersome the computations are, and the more suitable they would be for computer algebra.

The second, more algorithmic method was used in two different ways. If the density $\rho$ is known, we found the associated flux by splitting $D_{t} \rho$ into a piece that lies in the image of the operator $\Delta$ and a piece that does not. Sophisticated use of splitting technique allowed us to simultaneously find densities and fluxes at increased computational effort. The method was illustrated for the $\mathrm{mV}$ lattice.

A homotopic deformation of the KvM lattice was constructed. Further restriction of the deformation lead to the Miura map which links the KvM and $\mathrm{mV}$ lattices. Although cumbersome, recursive use of the Miura map allowed us to generate densities of the KvM lattice order by order.

MH wishes to thank the Department of Mathematical and Computer Sciences of the Colorado School of Mines for its hospitality during his sabbatical visit.

WH gratefully acknowledges the support of the National Science Foundation. This effort was sponsored in part under Grant CCR-9901929. 


\section{References}

Adler, V. E., Svinolupov, S. I. \& Yamilov, R. I. 1999 Multi-component Volterra and Toda type integrable equations. Phys. Lett. A 254, 24-36.

Adler, V. E., Shabat, A. B. \& Yamilov, R. I. 2000 Symmetry approach to the integrability problem. Theor. Math. Phys. 125, 1603-1661.

Cherdantsev, I. Yu. \& Yamilov, R. I. 1995 Master symmetries for differential-difference equations of the Volterra type. Physica D 87, 140-144.

Cherdantsev, I. \& Yamilov, R. 1996 Local master symmetries of differential-difference equations. In Symmetries and Integrability of Difference Equations (eds Levi, D., Vinet, L. \& Winternitz, P.). CRM Proceedings and Lecture Notes 9, pp. 51-61.

Clarkson, P. A. \& Nijhoff, F. W. 1999 Symmetries and Integrability of Difference Equations, London Mathematical Society Lecture Lecture Note Series, no. 255.

Fermi, E., Pasta, J. \& Ulam, S. 1965 Collected papers of Enrico Fermi II. University of Chicago Press, Chicago, Illinois. 978.

Göktaş, Ü. \& Hereman, W. 1997 Symbolic computation of conserved densities for systems of nonlinear evolution equations. J. Symb. Comp. 24, 591-621.

Göktaş, Ü. \& Hereman, W. 1998 Computation of conserved densities for nonlinear lattices. Physica D 123, 425-436.

Göktaş, Ü. \& Hereman, W. 1999 Algorithmic computation of higher-order symmetries for nonlinear evolution and lattice equations, Adv. Comp. Math. 11, 55-80.

Göktaş, Ü., Hereman, W. \& Erdmann, G. 1997 Computation of conserved densities for systems of nonlinear differential-difference equations. Phys. Lett. A 236, 30-38.

Hereman, W. 1996 Symbolic Software for Lie Symmetry Analysis. In CRC Handbook of Lie Group Analysis of Differential Equations Volume 3: New Trends in Theoretical Developments and Computational Methods (ed. N. H. Ibragimov) CRC Press, Boca Raton, Florida, pp. 367-413.

Hereman, W. \& Göktaş, Ü. 1999 Integrability Tests for Nonlinear Evolution Equations. In Computer Algebra Systems: A Practical Guide (ed. M. Wester). Wiley and Sons, New York, pp. 211-232.

Hereman, W., Göktaş, Ü., Colagrosso, M. \& Miller, A. 1998 Algorithmic integrability tests for nonlinear differential and lattice equations., Comp. Phys. Comm. 115, 4280-446.

Hietarinta, J., Nijhoff, F. W. \& Satsuma, J. eds, 2001 Symmetries and Integrability of Difference Equations. Special Issue dedicated to the SIDE IV Meeting, Tokyo, Japan, 2000. J. Phys. A: Math. Gen. 34 10337-10 737.

Hirota, R. \& Satsuma, J. 1976 N-soliton solution of nonlinear network equations describing a Volterra system. J. Phys. Soc. Jpn. 40, 891-900.

Kac, M. \& van Moerbeke, P. 1975 On an explicitly soluble system of nonlinear differential equations related to certain Toda lattices. Adv. Math. 16, 160-169.

Levi, D. \& Ragnisco, O. 1978 Extension of the spectral transform method for solving nonlinear differential-difference equations, Lett. Nuovo Cimento 22, 691-696.

Levi, D. \& Ragnisco, O. (eds) 2000 SIDE III - Symmetries and Integrability of Difference Equations., Meeting SIDE III, Sabaudia, Italy, 1998. CRM Proceedings and Lecture Notes 25. American Mathematical Society, Providence, Rhode Island.

Levi, D., Vinet, L. \& Winternitz, P. (eds) 1996 Symmetries and Integrability of Difference Equations. Meeting SIDE I, Esterel, Québec, Canada, 1994. CRM Proceedings and Lecture Notes 9. American Mathematical Society, Providence, Rhode Island.

Levi, D. \& Yamilov, R. I. 1997 Conditions for the existence of higher symmetries of evolutinary equations on the lattice. J. Math. Phys. 38, 6648-6674.

Manakov, S. 1975 Complete integrability and stochatizations of discrete dynamical systems. Sov. Phys. JETP 40, 269-274. 
Mikhailov, A. V., Shabat, A. B. \& Yamilov, R. I. 1987 The symmetry approach to the classification of nonlinear equations. Complete lists of integrable systems. Uspekhi Mat. Nauk 24, 3- 53. (Engl. Transl.: Russian Math. Surveys 42 1-63.)

Mikhailov, A. V., Shabat, A. B. \& Sokolov, V. V. 1990 The symmetry approach to classification of integrable equations, In What is Integrability? (ed. V. E. Zakharov). Springer Series in Nonlinear Dynamics, pp. 115-184.

Miura, R. M. 1968 Korteweg-de Vries equation and generalizations. I. A remarkable explicit non-linear transformation. J. Math. Phys. 9, 1202-1204.

Miura, R. M., Gardner, C. S. \& Kruskal, M. D. 1968 Korteweg-de Vries equation and generalizations. II. Existence of conservation laws and constants of motion. J. Math. Phys. 9, 1204-1209.

Moser, J. 1975 Three integrable Hamiltonian systems connected with isospectral deformations. Adv. Math. 16, 197-220.

Oevel, W., Zhang, H. \& Fuchssteiner, B. 1989 Master symmetries and multi-Hamiltonian formulations for some integrable lattice systems. Prog. Theor. Phys. 81, 294-308.

Ruijsenaars, S. N. M. 1990 Relativistic Toda systems. Comm. Math. Phys. 133, 217-247.

Shabat, A. B. \& Yamilov, R. I. 1988 Lattice representations of integrable systems. Phys. Lett. A 130, 271-275.

Shabat, A. B. \& Yamilov, R. I. 1991 Symmetries of nonlinear chains. Leningrad Math. J. 2, 377-400.

Shabat, A. B. \& Yamilov, R. I. 1997 To a transformation theory of two-dimensional integrable systems. Phys. Lett. A 227, 15-23.

Sokolov, V. V. \& Shabat, A. B. 1984 Classification of integrable evolution equations. Sov. Scient. Rev. C, Math. Phys. Rev. 4, 221-280.

Suris, Yu. B. 1997a New integrable systems related to the relativistic Toda lattice. J. Phys. A: Math. Gen. 30, 1745-1761.

Suris, Yu. B. $1997 b$ On some integrable systems related to the Toda lattice. J. Phys. A: Math. Gen. 30, 2235-2249.

Suris, Yu. B. 1999 Integrable discretizations for lattice systems: local equations of motion and their Hamiltonian properties. Rev. Math. Phys. 11, 727-822.

Suris, Yu. B. 2001 Miura transformations for Toda-type integrable systems, with applications to the problem of integrable discretizations. Sfb288 Preprint 367, Department of Mathematics, Technical University Berlin, Berlin, Germany.

Suris, Yu. B. 2002 The problem of integrable discretization: Hamiltonian approach. A skeleton of the book. Sfb288 Preprint 479, Department of Mathematics, Technical University Berlin, Berlin, Germany.

Svinolupov, S. I. \& Yamilov, R. I. 1991 The multi-field Schrödinger lattices. Phys. Lett. A 160, 548-552.

Teschl, G. 2000 Jacobi Operators and Completely Integrable Nonlinear Lattices. AMS Mathematical Surveys and Monographs 72. American Mathematical Society, Providence, Rhode Island.

Toda, M. 1989 Theory of Nonlinear Lattices, 2nd enlarged edition. Berlin: Springer Verlag.

Volterra, V. 1931 Leçons sur la théorie mathématique de la lutte pour la vie. Paris: Gauthier-Villars.

R. I. Yamilov, R. I. 1993 Classification of Toda type scalar lattices. In Proc. 8th Int. Workshop on Nonlinear Evolution Equations and Dynamical Systems, NEEDS'92, Dubna, U.S.S.R., 1992 (eds V. Makhankov, I. Puzynin, and O. Pashaev). World Scientific, Singapore, pp. 423-431.

Yamilov, R. I. 1994 Construction scheme for discrete Miura transformation. J. Phys. A: Math. Gen. 27, 6839-6851. 